\title{
Strain Tunable Optics of Elastomeric Microlens Array
}

\author{
Zhengwei Li and Jianliang Xiao*
}

Department of Mechanical Engineering, University of Colorado Boulder, Boulder, Colorado 80309, USA

*Email: iianliang.xiao@colorado.edu 
Microlens arrays (MLAs) have attracted great attention with their important applications in many fields, such as optical communications, organic light emitting diodes (OLEDs), thin film photovoltaic cells, and novel bio-inspired devices. And the dynamically tunable MLAs whose optical properties can be changed in a controllable manner is especially desirable in some recent developments. In this study, a very simple tuning method via mechanical stretching is proposed to tune the optics of elastomeric MLAs. Theoretical mechanics and optics studies are combined to demonstrate the tunability of elastomeric MLAs under both uniaxial and equibiaxial stretching. The results show that extremely large tuning range of the focal length can be achieved using this method. This theoretical study can provide important implications to not only the design of tunable MLAs, but also developments that require controllable optical elements.

Keywords:

Microlens array, Tunable optics, Mechanics, Ray tracing 


\section{Introduction}

Optical microlens arrays (MLAs) have been playing important roles in many fields, such as photolithography [1], optical communication [2], organic light emitting diodes (OLEDs) [3], thin film organic photovoltaic cells $[4,5]$, and biomimetic artificial compound eye cameras $[6,7]$. Some recent advancement in photonics and optoelectronics [8-14] requires tunability of the optics of MLAs, and therefore, a lot of research has been invested to realize tunable MLAs. Different tuning mechanisms, such as strain [15], stimuli-responsive hydrogels [16, 17], electrowetting [18], hydraulics [14, 19], dielectric [20] and electromagnetic actuation [21], have been utilized to change either microlens shape or refractive index [22] to realize tunable optics. However, among these developments, either costly fabrication and assembly processes are required, or only small tuning range can be provided.

In this paper, we demonstrate a very simple but effective tuning mechanism via mechanical stretching the elastomeric MLAs to tune the optical properties. Very large tuning range can be realized through this method. Figure 1a shows the schematic illustration of the tuning mechanism of elastomeric MLAs via mechanical stretching. The original MLA is composed of a square array of hemispherical elastomeric polydimethylsiloxane (PDMS, Sylgard 184) microlenses. Each microlens has the radius of curvature $0.4 \mathrm{~mm}$, and the distance between neighbor microlenses is $0.92 \mathrm{~mm}$. Under equibiaxial stretching, the shape of each microlens becomes more oblate but keeps axisymmetric and hemispherical. Under uniaxial stretching, each microlens is stretched along the tensile direction, but compressed in the lateral direction. The shape of each microlens surface changes from hemispherical to ellipsoidal. Therefore, each microlens possesses different focusing power along different directions [7, 23]. Figure $1 \mathrm{~b}$ shows the schematic illustration of the ray tracing model for optical simulations. To simplify the optical model, the thickness of the substrate 
is assumed to be large enough so that light rays converge inside the elastomeric medium. Although this simplification might not comply with some applications, it's sufficient to demonstrate the effectiveness of the tuning mechanism. Modifications to the optical model can be easily done for cases when the focal point falls outside of the elastomeric medium. When subject to stretching, the shapes of microlenses change, and their focal lengths change accordingly.

In this work, the deformation of the microlens under both equibiaxial and uniaxial stretching is analyzed by using finite element analysis (FEA). The surface profile of the deformed microlens is then extracted from the FEA model and implemented in the optical ray tracing simulation to study the change in optical properties. Then the height of the original hemispherical microlens is reduced to demonstrate even greater tunability in optics. This study can have important implications for not only the design of tunable MLAs, but also other developments that require controllable optical elements.

\section{Results and discussions}

\subsection{Mechanics of the tunable microlens}

Deformation of the microlens is studied when the MLA is subject to both equibiaxial and uniaxial stretching. Finite element analysis (FEA) is adopted to simulate microlens mechanics, by using software ABAQUS. Due to the periodic nature of the MLA, one unit cell that contains a hemispherical microlens and base membrane is used for FEA simulation. Periodic boundary conditions are applied to the side walls of the base membrane. The FEA model is discretized by eight-node, hexahedral solid elements. The whole unit cell is made of PDMS, which can be characterized by Yeoh hyperelastic model [24]. The strain energy density is given by 


$$
W=\sum_{k=1}^{3} C_{\mathrm{k}}\left(I_{1}-3\right)^{k}
$$

where $I_{1}=\lambda_{1}^{2}+\lambda_{2}^{2}+\lambda_{3}^{2}, \lambda_{1}, \lambda_{2}$ and $\lambda_{3}$ are the principal stretches, and $\lambda_{1} \lambda_{2} \lambda_{3}=1$ due to incompressible material behavior. The material constants are $C_{1}=0.285 \mathrm{MPa}, C_{2}=0.015 \mathrm{MPa}$ and $C_{3}=0.019 M P a[7,14]$.

Figure 2 shows the deformation and strain contours of the microlens unit cell under both equibiaxial and uniaxial tension. The maximum principal strain contours of the unit cell (upper frame) and the microlens (lower frame) when the MLA is subject to 100\% equibiaxial tensile strain are shown in Fig. 2a. The maximum principal strain in the microlens reaches $120 \%$ at the edge, but decays very quickly towards the center. In addition, the shape of the deformed microlens becomes oblate compared to the original hemisphere, but it remains axisymmetric. Figure $2 b$ presents the maximum principal strain in the microlens versus the equibiaxial tensile strain applied to the MLA. Results for the microlens unit cell subject to $100 \%$ uniaxial tensile strain are shown in Fig.2c. Along the tensile direction, the maximum principal strain (tensile) in the microlens reaches $114 \%$. Along the lateral direction, the microlens is under compression, due to Poisson's effect. The minimum principal strain (compressive) in the microlens in the lateral direction is $62 \%$. However, the strain decreases very quickly towards the center along both directions. Due to the asymmetric deformation, the shape of the deformed microlens changes from hemispherical to ellipsoidal. Figure $2 \mathrm{~d}$ shows the relationship between the maximum principal strain along the tensile direction (tensile) and the minimum principal strain along the lateral direction (compressive) versus the uniaxial tensile strain applied to the MLA. 


\subsection{Surface profile of the tunable microlens}

The change of the microlens surface profile is studied as it directly affects the optical properties [7]. Under equibiaxial tension, the shape of the microlens surface keeps hemispherical but becomes more oblate, as demonstrated in Fig. 2a. Figure 3a shows the microlens surface profile extracted from FEA simulation when the MLA is subject to $20 \%$ equibiaxial tensile strain. The profile can be fitted very well by a circular curve with radius of curvature $0.42 \mathrm{~mm}$. The method of least squares was adopted to conduct the profile fitting. Due to the severe distortion around the edge as shown in Figs. 2a, data near the edge (distance to the central axis $>0.3 \mathrm{~mm}$ ) were excluded from the fitting. This treatment doesn't affect the applicability of the results, as the central part of the microlens is more important for focusing and an aperture can always be added to block the periphery area. The microlens profile from FEA simulation when the MLA is subject to $100 \%$ equibiaxial tensile strain is presented in Fig. 3b, which can be fitted very well by a circular curve of radius $0.7 \mathrm{~mm}$. The radius of curvature of the tunable microlens normalized by the original radius versus the applied equibiaxial tensile strain is shown in Fig. 3c. The radius of the microlens increases by $75 \%$ when $100 \%$ equibiaxial tensile strain is applied.

When the MLA is subject to uniaxial stretching, the shape of the microlens surface changes from hemispherical to ellipsoidal, as demonstrated in Fig. 2c due to the Poisson's effect. Figure $3 \mathrm{~d}$ shows the surface profiles along both tensile ( $\mathrm{x}$ direction, major axis) and lateral (y direction, minor axis) directions when $20 \%$ uniaxial tension is applied to the MLA. Both profiles can be

fitted very well by circular curves of radii $R_{\mathrm{x}}=0.43 \mathrm{~mm}$ and $R_{\mathrm{y}}=0.38 \mathrm{~mm}$, respectively. Similar to equibiaxial stretching, only central part of the microlens with height measured from the base membrane larger than $0.25 \mathrm{~mm}$ are used for fitting. The microlens surface profiles and corresponding fitting curves when the MLA is subject to $100 \%$ uniaxial tension are given in Fig. 
3e. The fitting gives the radii of the surface profiles along both major and minor axes as $R_{\mathrm{x}}=0.66 \mathrm{~mm}$ and $R_{\mathrm{y}}=0.33 \mathrm{~mm}$, respectively. Figure $3 \mathrm{f}$ shows the normalized radii of curvature of the microlens surface profiles along both major and minor axes versus the applied uniaxial tensile strain. The radius of the surface profile along major axis increases by $64 \%$ and that along the minor axis decreases by $19 \%$ when $100 \%$ uniaxial tension is applied.

\subsection{Optics of the tunable microlens}

The focal length is one of the most optical parameters of a microlens. For paraxial light rays, the focal length of the microlens can be given as [25]

$$
f=\frac{r \cdot n}{n-1},
$$

where $r$ is the radius of curvature of the microlens surface, $\mathrm{n}=1.43$ is the refractive index of PDMS $[6,7]$. This equation, however, doesn't works when the light beam is large, i.e. light rays can be distant away from the optical axis of the microlens [7]. In this work, the focal lengths of microlenses are determined by ray tracing simulations using FRED optical software (Photon Engineering LLC). The fitted surface profiles from the previous section are used to build microlenses in the optical model. Collimated light beam of radius $0.2 \mathrm{~mm}$ is adopted for all ray tracing simulations.

Figure 4a shows the relationship between the focal length of the tunable microlens (normalized by the focal length of the undeformed microlens) and the equibiaxial tensile strain. Due to stretching, the microlens surface changes from hemispherical to oblate, and the radius of curvature increases. This leads to increasing focal length of the microlens. When $100 \%$ equibiaxial 
stretching is applied to the MLA, the focal length of the microlens increases by $81 \%$. Figure $4 \mathrm{~b}$ shows the normalized focal lengths of the tunable microlens along major and minor axes versus the applied uniaxial tensile strain. When the MLA is stretched uniaxially, the shape of the microlens changes from hemispherical to ellipsoidal, whose optical property can be characterized by two focal lengths, $f_{\mathrm{x}}$ along the major axis and $f_{\mathrm{y}}$ along the minor axis. Due to stretching along the major axis, the surface profile becomes more oblate, and the focal length increases. On the contrary, the microlens is under compression along the minor axis, the surface profile becomes more prolate, and the focal length decreases. As shown in Fig. $4 \mathrm{~b}$, the focal length $f_{\mathrm{x}}$ along the major axis increases by $69 \%$ and the focal length $f_{\mathrm{y}}$ along the minor axis decreases by $20 \%$, when $100 \%$ uniaxial tensile strain is applied to the MLA.

As indicated by the previous results, the tunability of the microlens strongly depends on how much the surface profile changes when the MLA is mechanically stretched. Since reducing the height of the hemispherical microlens can effectively enhance the degree of surface profile change when subject to stretching, similar mechanics and optics simulations have been performed for a spherical cap whose height is only $1 / 3$ of the radius, as illustrated by the inset of Fig. 4c. Figure $4 \mathrm{c}$ shows the normalized focal length of the microlens versus the applied equibiaxial tensile strain. When the MLA is subject to $100 \%$ equibiaxial tension, the focal length of the microlens increases by $460 \%$. The tuning range of this $1 / 3$ hemispherical cap microlens is 5.75 times of the tuning range of the full hemispherical microlens. Figure $4 \mathrm{~d}$ shows the normalized focal lengths along major and minor axes, $f_{\mathrm{x}}$ and $f_{\mathrm{y}}$, versus the applied uniaxial tensile strain. When $100 \%$ uniaxial tension is applied to the MLA with $1 / 3$ hemispherical cap microlens, the focal length along major axis $f_{\mathrm{x}}$ increases by $226 \%$, which is 3.28 times of that of a full hemispherical microlens. 
The focal length along the minor axis $f_{\mathrm{y}}$ decreases by $31 \%$, which is 1.55 times of that of a full hemispherical microlens.

\section{Conclusions}

In this study, a very simple but effective mechanism via mechanical stretching to tune the optics of elastomeric MLAs is proposed and investigated. Mechanics and optics of tunable PDMS MLAs under both equibiaxial and uniaxial tension are studied. Results show that extremely large tunability of the microlens optics can be realized by this method. When full hemispherical microlenses are adopted, 100\% equibiaxial stretching yields $80 \%$ increase in focal length, and $100 \%$ uniaxial stretching leads to $69 \%$ increase in focal length along major axis and $20 \%$ decrease in focal length along minor axis. Partial hemispherical cap microlenses can further enhance the tunability. For a 1/3 hemispherical cap microlens, 100\% equibiaxial stretching yields $460 \%$ increase in focal length, and $100 \%$ uniaxial stretching leads to $226 \%$ increase in focal length along major axis and $31 \%$ decrease in focal length along minor axis. This theoretical study can provide important information for designing tunable MLAs or systems that require controllable optical elements.

\section{Acknowledgements}

The authors gratefully acknowledge support from ACS Petroleum Research Fund (Grant No. 53780-DN17) and NSF (Grant No. CMMI-1405355). 


\section{References}

[1] R. Vo, H.P. Herzig, P. Nussbaum and W.B. Hugle. Microlens array imaging system for photolithography. Optical Engineering 35, no. 11 (1996): 3323-3330.

[2] N. Chronis, G. Liu, K.H. Jeong and L. Lee. Tunable liquid-filled microlens array integrated with microfluidic network. Optics Express 11, no. 19 (2003): 2370-2378.

[3] E. Wrzesniewski, S.H. Eom, W. Cao, W.T. Hammond, S. Lee, E.P. Douglas and J. Xue. Enhancing Light Extraction in Top - Emitting Organic Light - Emitting Devices Using Molded Transparent Polymer Microlens Arrays. Small 8, no. 17 (2012): 2647-2651.

[4] K. Tvingstedt, S Dal Zilio, O. Inganńs and M. Trapping light with micro lenses in thin film organic photovoltaic cells. Optics Express 16, no. 26 (2008): 21608-21615.

[5] Y. Chen, M. Elshobaki, Z. Ye, J.M. Park, M.A. Noack, K.M. Ho and S. Chaudhary. Microlens array induced light absorption enhancement in polymer solar cells. Physical Chemistry Chemical Physics15, no. 12 (2013): 4297-4302.

[6] Y. M. Song, Y. Xie, V. Malyarchuk, J. Xiao, I. Jung, K. J. Choi, Z. Liu, H. Park, C. Lu, R. H. Kim, R. Li, K. B. Crozier, Y. Huang, and J. A. Rogers. Digital cameras with designs inspired by the arthropod eye. Nature 497, no. 7447 (2013): 95-99.

[7] Z. Li and J. Xiao. Mechanics and optics of stretchable elastomeric microlens array for artificial compound eye camera. Journal of Applied Physics 117, no. 1 (2015): 014904.

[8] G. Beadie, M. L. Sandrock, M. J. Wiggins, R. S. Lepkowicz, J. S. Shirk, M. Ponting, Y. Yang, T. Kazmierczak, A. Hiltner and E. Baer. Tunable polymer lens. Optics express 16, no. 16 (2008): 1184711857.

[9] X. Xu, M. Davanco, X. Qi and S.R. Forrest. Direct transfer patterning on three dimensionally deformed surfaces at micrometer resolutions and its application to hemispherical focal plane detector arrays. Organic Electronics 9, no. 6 (2008): 1122-1127.

[10] Z. Li, Y. Wang and J. Xiao. Mechanics of curvilinear electronics and optoelectronics. Current Opinion in Solid State and Materials Science (2015).

[11] D. Kang, S.M. Lee, Z. Li, A. Seyedi, J. O'Brien, J. Xiao, and J. Yoon. Compliant, Heterogeneously Integrated GaAs Micro - VCSELs towards Wearable and Implantable Integrated Optoelectronics Platforms. Advanced Optical Materials 2, no. 4 (2014): 373-381.

[12] H. C. Ko, M. P. Stoykovich, J. Song, V. Malyarchuk, W. M. Choi, C. J. Yu, J. B. Geddes, J. Xiao, S. Wang, Y. Huang, and J. A. Rogers. A hemispherical electronic eye camera based on compressible silicon optoelectronics. Nature 454, no. 7205 (2008): 748-753.

[13] S. Wang, J. Xiao, I. Jung, J. Song, H.C. Ko, M.P. Stoykovich, Y. Huang, K.C. Hwang and J.A. Rogers. Mechanics of hemispherical electronics. Applied Physics Letters 95, no. 18 (2009): 181912. 
[14] I. W. Jung, J. Xiao, V. Malyarchuk, C. Lu, M. Li, Z. Liu, J. Yoon, Y. Huang and J. A. Rogers. Dynamically tunable hemispherical electronic eye camera system with adjustable zoom capability. Proceedings of the National Academy of Sciences 108, no. 5 (2011): 1788-1793.

[15] D. Chandra, S. Yang and P.C. Lin. Strain responsive concave and convex microlens arrays. Applied Physics Letters 91, no. 25 (2007): 251912.

[16] L. Dong, A.K. Agarwal, D. J. Beebe and H. Jiang. Adaptive liquid microlenses activated by stimuliresponsive hydrogels. Nature442, no. 7102 (2006): 551-554.

[17] L. Dong, A. K. Agarwal, D. J. Beebe and H. Jiang. Variable - Focus Liquid Microlenses and Microlens Arrays Actuated by Thermoresponsive Hydrogels. Advanced Materials 19, no. 3 (2007): 401-405.

[18] T. Krupenkin, S. Yang and P. Mach. Tunable liquid microlens. Applied Physics Letters 82, no. 3 (2003): 316-318.

[19] C. Lü, M. Li, J. Xiao, I. Jung, J. Wu, Y. Huang, K. C. Hwang and J. A. Rogers. Mechanics of tunable hemispherical electronic eye camera systems that combine rigid device elements with soft elastomers. Journal of Applied Mechanics 80, no. 6 (2013): 061022.

[20] F. Carpi, G. Frediani, S. Turco and D. De. Rossi. Bioinspired Tunable Lens with Muscle - Like Electroactive Elastomers. Advanced Functional Materials 21, no. 21 (2011): 4152-4158.

[21] P. Liebetraut, S. Petsch, W. Mönch, and H. Zappe. Tunable solid-body elastomer lenses with electromagnetic actuation. Applied optics 50, no. 19 (2011): 3268-3274.

[22] A. Llobera, R. Wilke, and S. Büttgenbach. Poly (dimethylsiloxane) hollow Abbe prism with microlenses for detection based on absorption and refractive index shift. Lab on a Chip 4, no. 1 (2004): 2427.

[23] D.B. Do, N.D. Lai, C.Y. Wu, J.H. Lin and C.C. Hsu. Fabrication of ellipticity-controlled microlens arrays by controlling the parameters of the multiple-exposure two-beam interference technique. Applied optics 50, no. 4 (2011): 579-585.

[24] O.H.Yeoh. Some forms of the strain energy function for rubber. Rubber Chemistry and technology 66, no. 5 (1993): 754-771.

[25] E. Hecht, and A. Zajac, Optics (Addison Wesley, New York, 2002). 


\section{Figure captions}

Fig. 1. Schematic illustration of the tuning mechanism of elastomeric microlens arrays (MLAs) via mechanical stretching (a) and the ray tracing simulation model (b).

Fig. 2. Mechanics of tunable microlens unit cell under equibiaxial and uniaxial tension. (a) Maximum principal strain contours of a microlens unit cell (upper frame) and a microlens (lower frame) under $100 \%$ equibiaxial tensile strain, (b) maximum principal strain in the microlens versus the applied equibiaxial tensile strain, (c) maximum principal strain contours of a microlens unit cell (upper frame) and a microlens (lower frame) under 100\% uniaxial tensile strain, (d) maximum principal strain (tensile) along the tensile direction and minimum principal strain (compressive) along the lateral direction in the microlens versus the applied uniaxial tensile strain.

Fig. 3. Surface profiles of the tunable microlens under equibiaxial and uniaxial tensile strains. The surface profiles of the microlens under $20 \%$ (a) and $100 \%$ (b) equibiaxial tensile strains, (c) the radius of curvature of the tunable microlens (normalized by the radius of the undeformed microlens) versus the tensile strain when the MLA is subject to equibiaxial tension; the surface profiles of the microlens under $20 \%$ (d) and $100 \%$ (e) uniaxial tensile strains, (f) the normalized radii of curvature of the tunable microlens along major and minor axes versus the tensile strain when the MLA is subject to uniaxial tension. 
Fig. 4. Optics of the tunable microlens under equibiaxial and uniaxial tensile strains. The relationship between the focal length of a full hemispherical microlens (normalized by the focal length of the undeformed microlens) and the tensile strain under equibiaxial tension (a) and uniaxial tension (b). For a 1/3 hemispherical cap microlens, the relationship between the normalized focal length and the tensile strain under equibiaxial tension (c) and uniaxial tension (d). 
Figure 1. Schematic illustration

a)

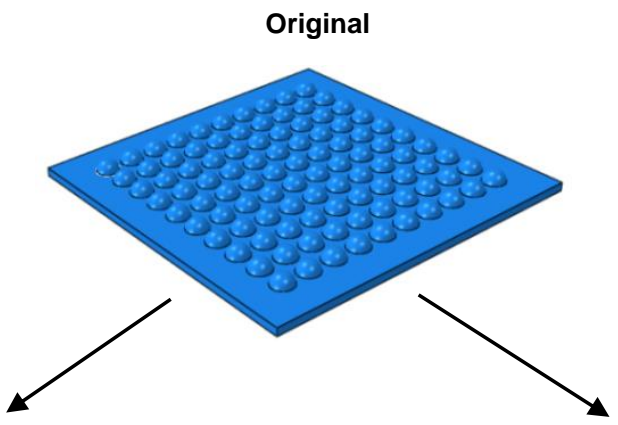

Equibiaxial stretching

Uniaxial stretching
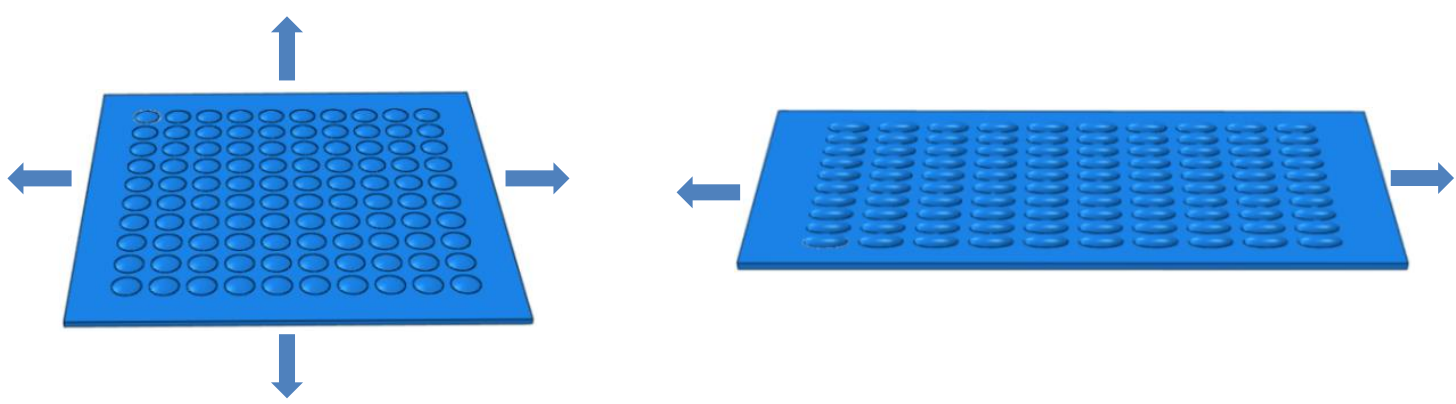

b)

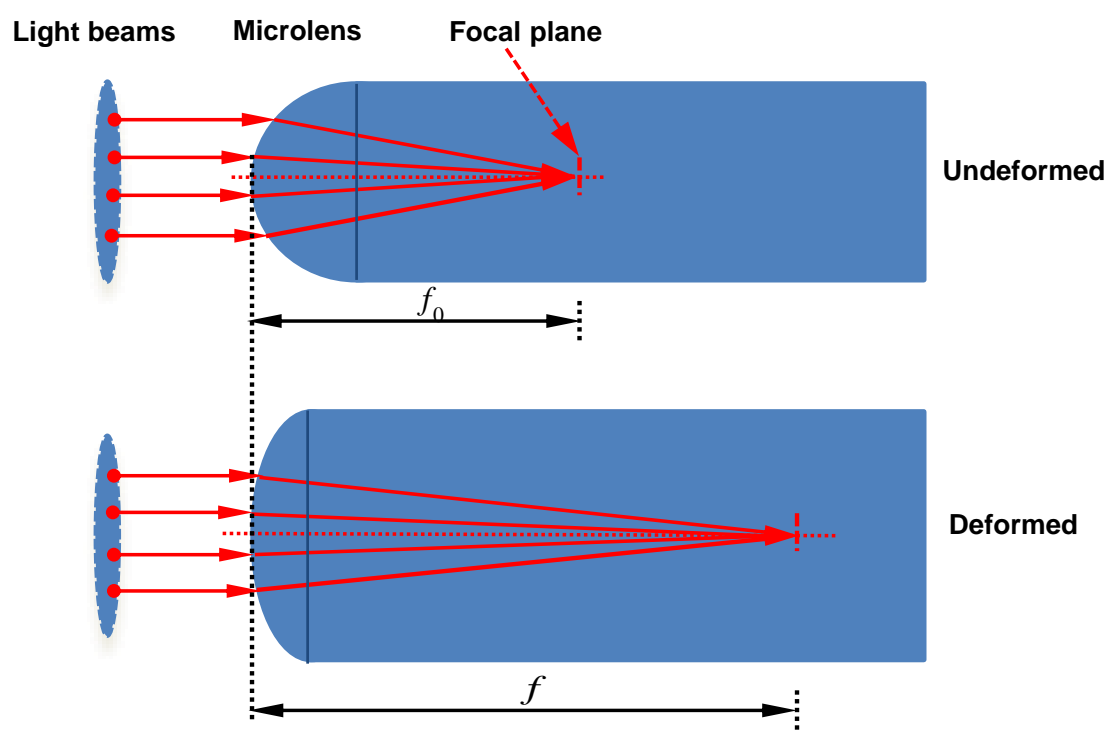


Figure 2. Mechanics

a)

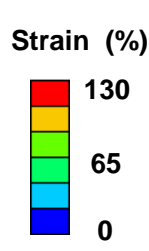

Strain (\%)

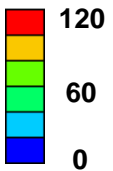

b)
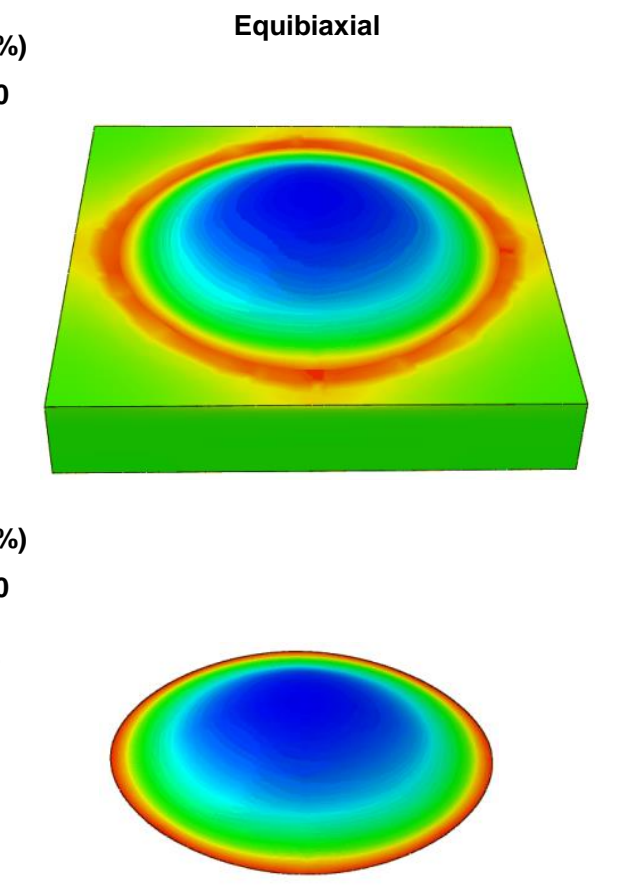

Equibiaxial

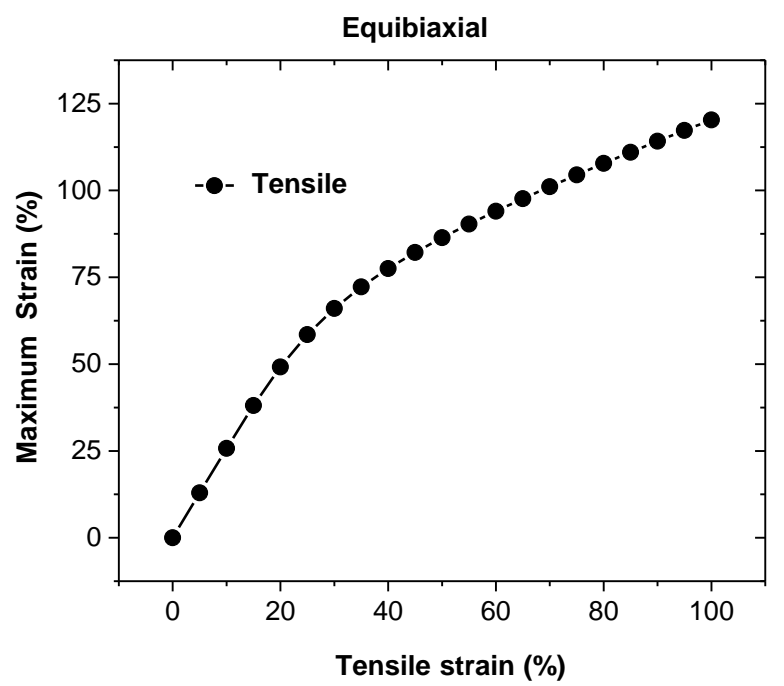

c)

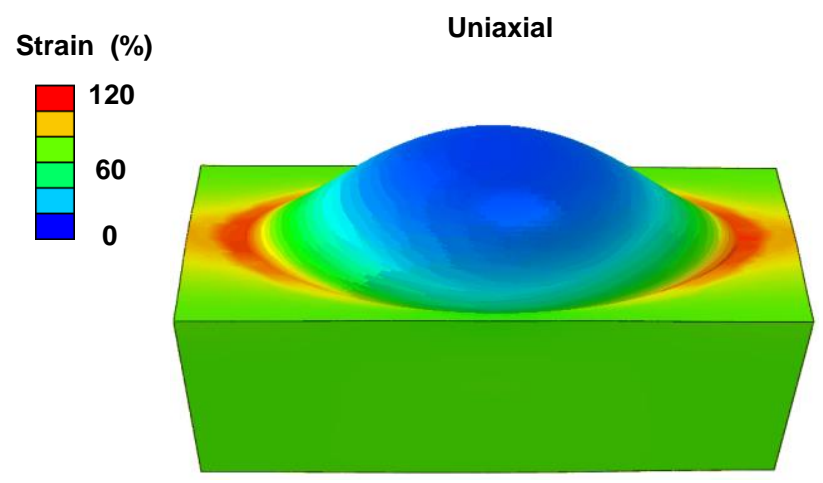

Strain (\%)

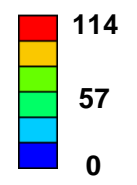

d)

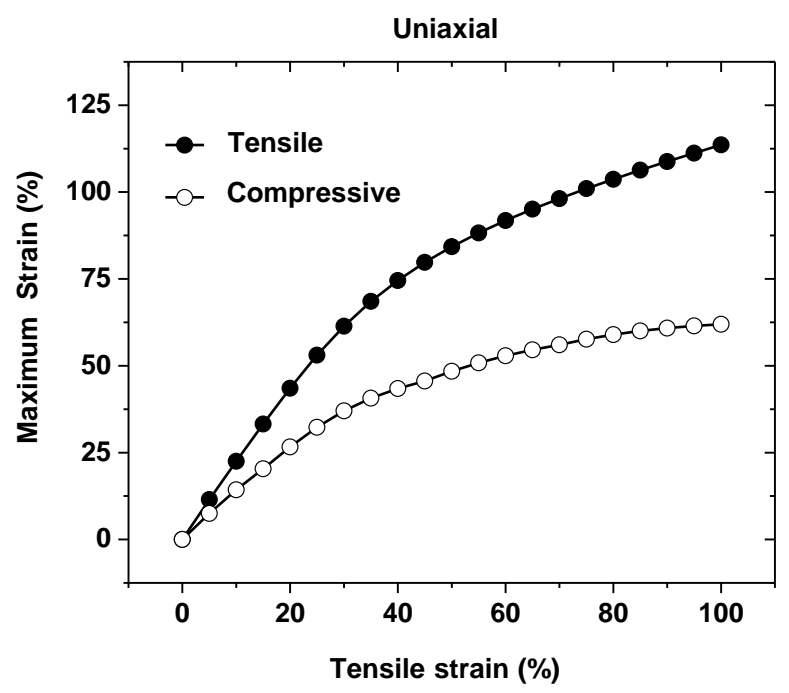


Figure 3. Surface Profiles

a)



b)

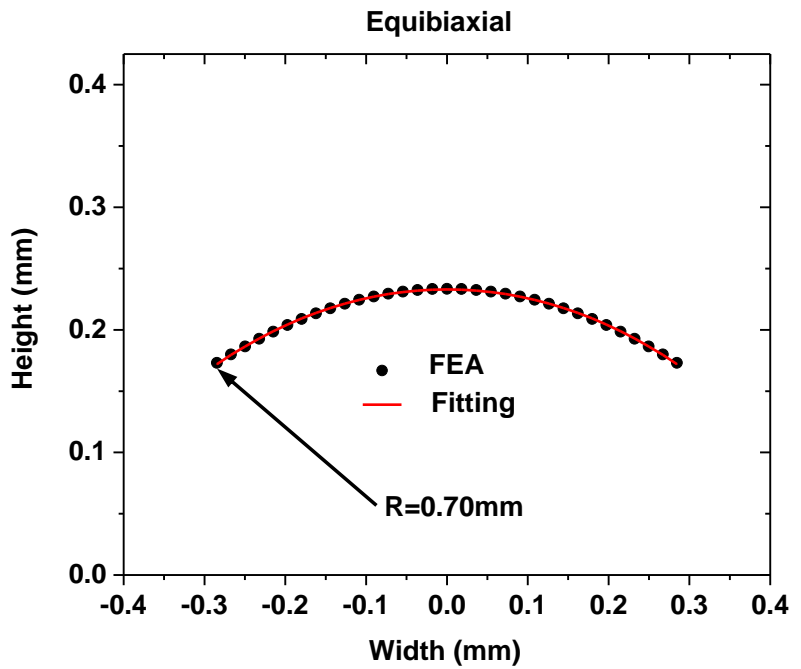

c)

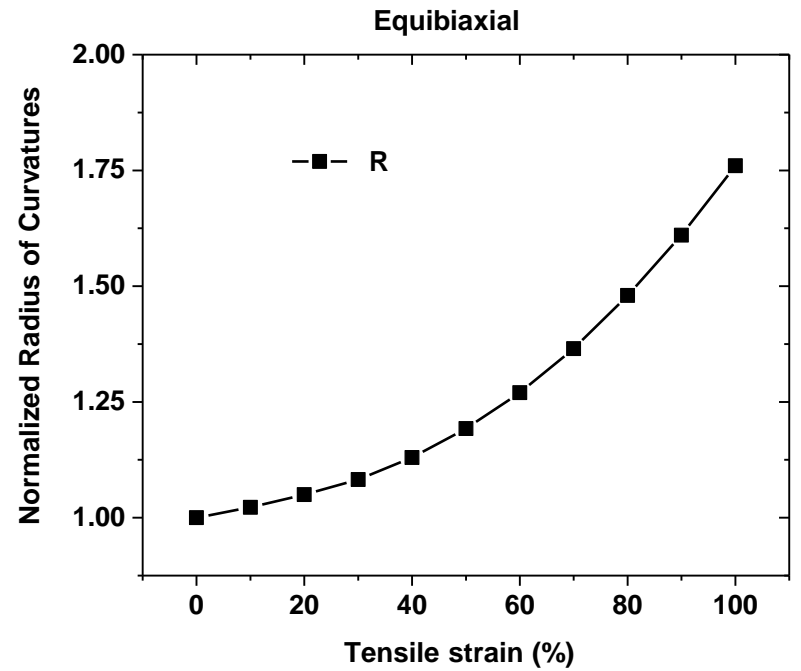

d)

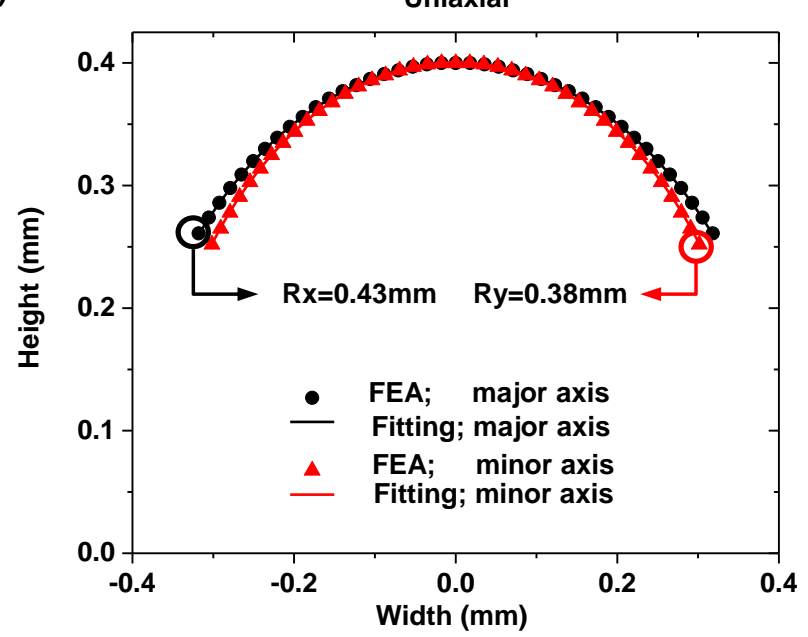

e)

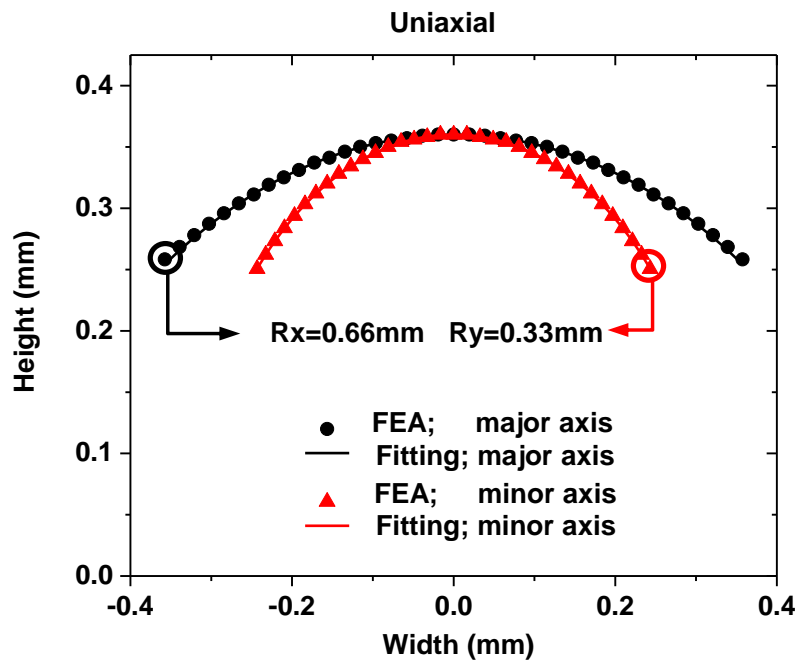

f)

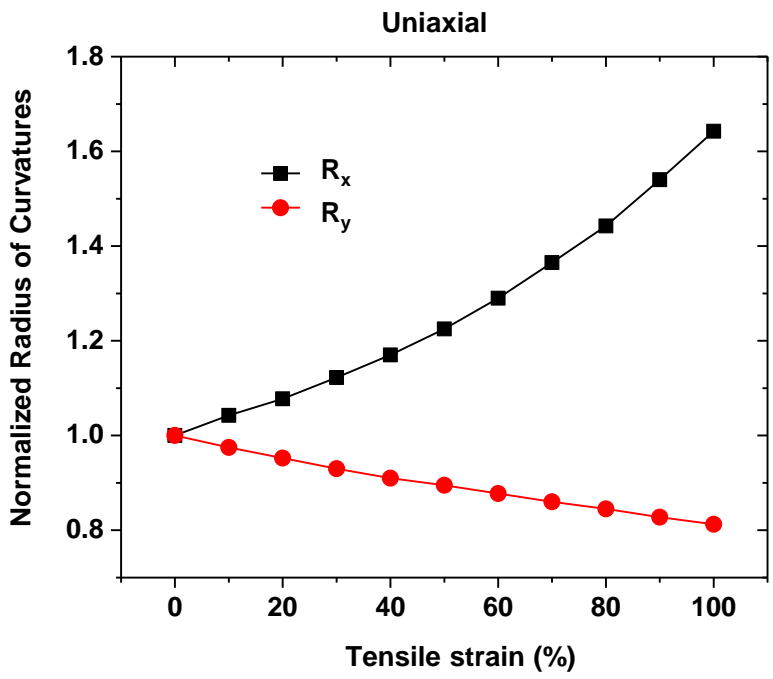


a)

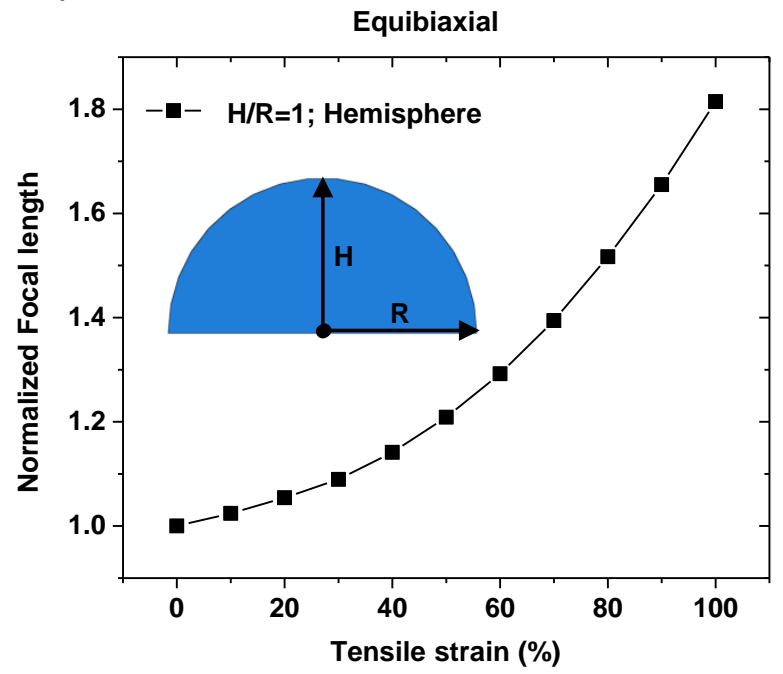

c)

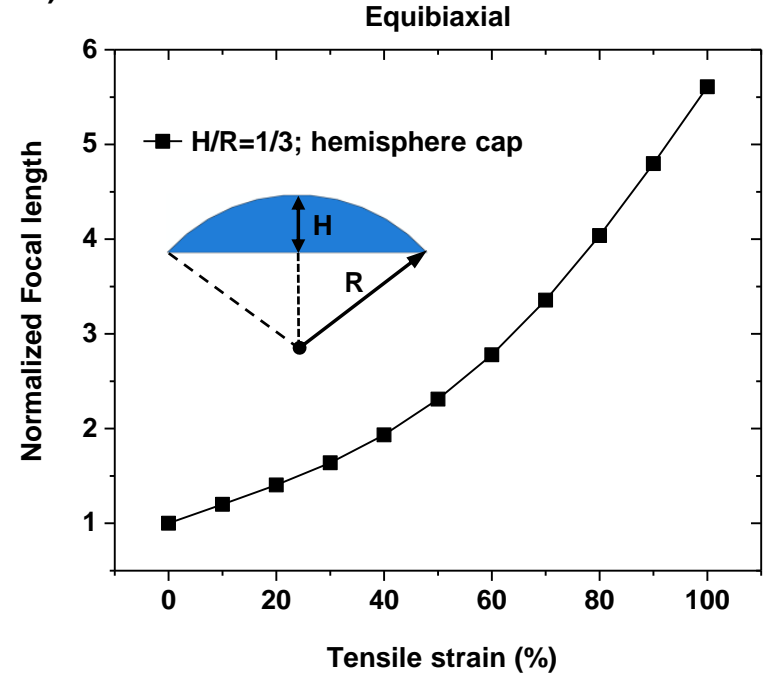

b)

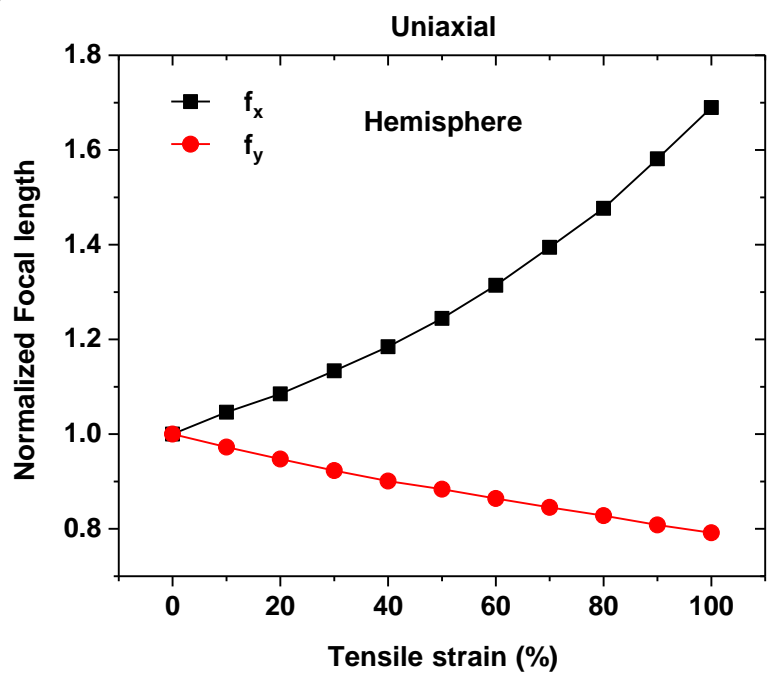

d)

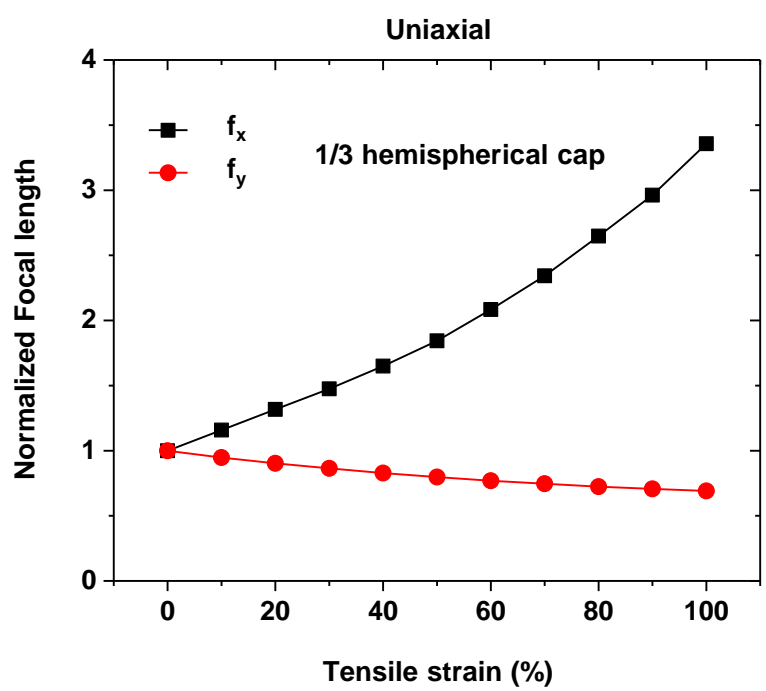

